SN: $08 / 307,981$

FD: $09.02-94$

Registration No. RB239530643

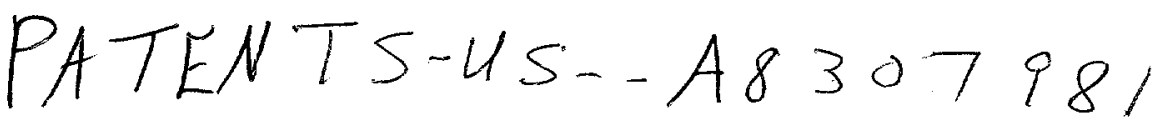

\title{
NUCLEAR RESONANCE TOMOGRAPHY WITH A TOROID CAVITY DETECTOR
}

Klaus Woelk Jerome W. Rathke Robert J. Klingler

\section{DISCLAIMER}

\begin{abstract}
This report was prepared as an account of work sponsored by an agency of the United States Government. Neither the United States Government nor any agency thereof, nor any of their employees, makes any warranty, express or implied, or assumes any legal liability or responsibility for the accuracy, completeness, or usefulness of any information, apparatus, product, or process disclosed, or represents that its use would not infringe privately owned rights. Reference herein to any specific commercial product, process, or service by trade name, trademark, manufacturer, or otherwise does not necessarily constitute or imply its endorsement, recommendation, or favoring by the United States Government or any agency thereof. The views and opinions of authors expressed herein do not necessarily state or reflect those of the United States Government or any agency thereof.
\end{abstract}
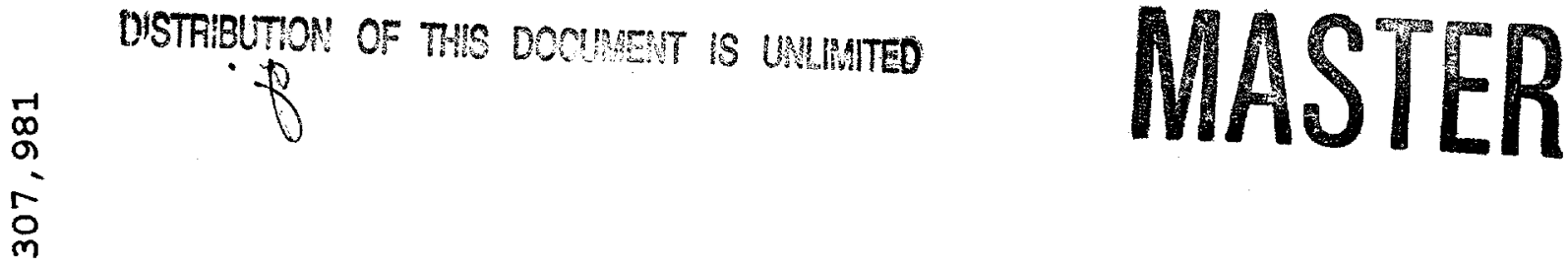


\section{DISCLAMMER}

Portions of this document may be illegible in electronic image products. Images are produced from the best available original document. 


\section{CONTRACTUAL ORIGIN OF THE INVENTION}

The United States Government has rights in this invention pursuant to Contract No. W-31-109-ENG-38 between the U.S. Department of Energy and the University of Chicago.

\section{BACKGROUND OF THE INVENTION}

This invention relates to an improved apparatus for nuclear magnetic resonance (NMR) imaging using a Rotating Frame Imaging (RFI) technique, and in particular, to a method and apparatus for NMR tomographic imaging of a sample to provide chemical shift information and micrometer distance resolution for the 10 sample. 
RFI employs a gradient in the radio-frequency field $\left(B_{1}\right)$ instead of a gradient in the static main magnetic field $\left(\mathrm{B}_{0}\right)$, where $\mathrm{B}_{1}$ is perpendicular to $\mathrm{B}_{0}$, to resolve distances associated with respective samples. A major advantage of this method is that the resonance frequency of the nuclear spins associated with the samples is not manipulated to measure the distance. Thus, through the implementation of RFI, associated chemical shifts and distances can be resolved simultaneously.

In the past, it has been difficult to create a $B_{1}$ gradient that is both mathematically well defined and strong enough to facilitate resolutions of distances below $0.1 \mathrm{~mm}$ using RFI. As a result, RFI was found to be unsuitable for NMR

10 microscopy. Now, however, the subject toroid cavity detector satisfies the requirements for implementation of rotating frame microscopy (RFM) to sample analysis.

When a high pressure environment is needed to conduct sample testing, a pressure vessel is used to contain the toroid cavity monitor and the NMR sample or 15 samples or, in the alternative, the toroid cavity monitor houses the sample which in turn is surrounded by the pressure vessel. The composite unit is placed in an externally applied homogeneous magnetic field $\left(B_{0}\right)$. The presence of $B_{0}$ causes the magnetic moments of a targeted class of nuclei in the samples to precess about the field's axis at a rate which is dependent on the magnetic field strength. A coil or 20 toroid internal to the pressure vessel is oriented in such a manner as to apply a magnetic field $\left(B_{1}\right)$ perpendicular to $B_{0}$, and then, the $B_{1}$ field is alternately energized and de-energized. First, the coil or toroid transmits or energizes, thereby shifting the 
precession of the magnetic moments away from the $\mathrm{B}_{0}$ axis, and second, the coil or toroid receives the sample's response as the magnetic moments subsequently return to their equilibrium values. In most applications, the received signal from the energized nuclei serves as the input signal for spectroscopic analysis of the sample. U.S. Patent 5,045,793 teaches the use of a free-standing, helical wound, toroidal coil as an rf detector for use with a high pressure NMR probe. In the subject invention, the transmitter/receiver coil is replaced by a cylindrical toroid cavity detector. In one embodiment of the present invention, the toroid cavity is a cylindrically shaped, hollow body made of copper with closed ends. A teflon coated

10 wire is fed through the base of the cylinder, and is positioned coincident with the major axis of the cylinder. In an alternate embodiment, slots are machined in the wall, top and bottom of the toroidal cylinder to provide access to the toroid cavity for a fluid or gas sample during the NMR experiment. In a further variation, a series of openings and sample holders positioned in the top of the toroidal cylinder provide

15 access for tubes containing the samples to the toroidal cavity detector or resonator.

Toroidal cavity resonators are known in the prior art, Alderman, however, the subject invention differs from the existing art in the method of tuning and in the structure of the toroid cavity resonator employed. In prior art, the toroid was tuned internally by means of the small gap in the cylindrical axis while the subject 20 invention is externally tuned and no gap exits in the teflon coated wire or central conductor which is oriented along the cylindrical axis. The present invention achieves significant results through the presence of a well-defined magnetic field $B_{1}$ 
gradient, which has been shown theoretically and experimentally to vary with the inverse of the radial displacement from the axial center of the cavity. As a result, the alternating magnetic field is strongest near the central conductor and drops off towards the outer wall of the toroidal cavity detector. The well-defined magnetic field provides a means to image simultaneously both the chemical shift of the targeted nuclei as well as their radial distance from the center.

The amount of energy that is absorbed/transmitted varies with the location of the sample within the toroidal cavity detector because the efficiency of the energy transfer process is proportional to $B_{1}$. By varying the transmitter pulse length,

10 multiple distances within the NMR active sample can be resolved. As a result, the major advantage of the new approach is that the chemical shift information is not destroyed by the imaging process as was the case when the measurement was conducted within a homogeneous static magnetic field, $\mathrm{B}_{0}$, by converting the chemical shift information to a measure of distance with a gradient in $\mathrm{B}_{0}$. Also, with 15 this invention, the shim system does not need to be altered.

The toroid cavity of the present invention, has several advantages over the prior art in that it provides a rugged reaction chamber that is readily machined from a variety of alloys, and the alternating magnetic field is highly confined within the cavity detector. Confining the alternating magnetic field minimizes sensitivity losses

20 that occur through magnetic coupling with a high-pressure housing. Most importantly, the cavity detector produces a well-defined magnetic field gradient, which, as noted previously, is predicted to vary with the inverse of the radial 
displacement from the center of the cavity. The resultant NMR intensity $\left(I / I_{1}\right)$ is predicted to depend on the transmitter pulse length, $t$, according to the following equation

$$
\mathrm{I} / \mathrm{I}_{0}=2 \pi \mathrm{h} \int \sin (\gamma \mathrm{At} / \mathrm{r}) \mathrm{dr}
$$

where $\gamma$ is the gyromagnetic ratio, $h$ is the height of the toroid, $r$ is the radial distance from the center of the cavity, and $\mathrm{A}$ is the proportionality constant defining the magnetic field as follows:

$$
\mathrm{B}_{1}=\mathrm{A} / \mathrm{r}
$$

The $1 / \mathrm{r}$ relation for the $B_{1}$ field suggests that both the NMR sensitivity and the

10 distance resolution should increase for materials that are close to the central conductor. Thus, the toroid cavity NMR resonator is envisioned to be particularly powerful in the characterization of surface layers applied to the central conductor. For example, two layers of tetraflouroethylene fluorocarbon resins were placed around the central conductor of the toroid cavity resonator. The measured thickness

15 of the respective layers was measured as well as their $20 \mu \mathrm{m}$ separation by means of the NMR imaging data.

Other potential uses for the toroid cavity resonator include in situ monitoring of growth sites during material formation. Additionally, it should be possible to monitor both the gas phase and the active surface sites during chemical vapor

20 deposition by heating the central conductor of the toroid cavity resonator. The toroid cavity detector can be used with a high pressure metal probe housing to conduct measurements at high pressures. Thus, a supercritical fluid at high pressure can be 
used as the solvent medium to accelerate the diffusion of viscous preceramic polymer reactants into permeable composite materials.

The toroid cavity detectors consist of an inner wire (central conductor) and an outer cylindrical body. With this arrangement, the $\mathrm{B}_{1}$ field is completely confined within the cavity. It is strongest near the central conductor and drops off as the inverse of the distance toward the outer wall. Both sensitivity and distance resolution increase with the gradient in $B_{1}$ and consequently, the toroid detector is well suited for NMR microscopy of films that uniformly surround the central conductor. In addition, the toroid cavity detectors appear to be useful for

10 investigations of solids and polymers where broad lines usually limit the spatial resolution to $50-100 \mu \mathrm{m}$. High-pressure and high-temperature capabilities of toroid cavities enable measurements of penetration rates in polymer or ceramic films in situ. In addition, electrochemical processes can be monitored, when the central conductor is used as a working electrode.

15 Since the $B_{1}$ field is strongest near the toroid's central conductor and drops off as the wall is approached, the amount of energy that is absorbed by any sample nuclei present varies with its location within the torus. Differences in the energy absorbed by the nuclei are manifest in their respective pulse rotation angles. Hence, the distances of various types of sample nuclei from the central conductor can be 20 resolved by determining their pulse rotation angles.

Conventional NMR imaging techniques sacrifice all of the spectral details as a side effect of the process used to encode distance information. In contrast, 
complete NMR spectral information is retained during signal processing with the toroid cavity imaging method. Additionally, because of the strong gradient that is intrinsic to the torus, the theoretical spacial resolution of the toroid cavity imager is better than is possible with conventional MRI. Also, spatial resolution with the toroid cavity imager is less dependent on the line widths of the NMR signals used in the measurements because chemical shift information is not used to determine the distance as it is in conventional MRI.

Rotating Frame Imaging (RFI) uses the $\mathrm{B}_{1}$ field gradient of an asymmetrically shaped NMR transmitter coil to achieve its spatial resolution. The

10 transmitted energy, and thus, the pulse rotation angle of the NMR active nuclei varies with the $B_{1}$ field strength. Accordingly, a gradient can be used for spin localization. Through incremental increases in the pulse width, a set of amplitude-modulated spectra is derived in which the modulation frequencies yield the spatial information. Using a two-dimensional Fourier transformation, both the spatial and the chemical15 shift information is resolved. This is a major advantage of the RFI technique over common MRI (Magnetic Resonance Imaging) where the chemical shift information is converted into a measure of distance by using a gradient in the main magnetic field $\mathrm{B}_{0}$.

NMR microscopy has always suffered from a lack of a $B_{1}$ field gradient that

20 is both accurate and strong enough to resolve distances less than $0.1 \mathrm{~mm}$. The toroid cavity detector of the subject invention satisfies RFM (rotating frame microscopy) 
requirements and facilitates resolution of distances at the micrometer level while simultaneously conserving the chemical-shift information.

Since, the $B_{1}$ field of toroid NMR detectors is completely confined within the torus, the use of a cavity instead of a coil to form the torus eliminates the irregularities found in the field of the coil due to the spaces between the individual turns of the coil.

It is the object of this invention to provide a NMR sampling probe which can determine the chemical shift of the targeted nuclei as well as their radial distance from the center.

It is a further object of this invention to teach a toroid cavity detector which is tuned externally.

Another object of this invention provides for sampling the targeted nuclei under high pressure conditions when coupled with a high pressure housing.

Additional advantages, objects and novel features of the invention will

15 become apparent to those skilled in the art upon examination of the following and by practice of the invention.

\section{SUMMARY OF THE INVENTION}

To achieve the foregoing and other advantages, this invention comprises a toroid cavity resonator consisting of an outer cylindrical body and an inner wire 20 located along the central axis of the cylindrical body. When energized, the cavity produces a $B_{1}$ field which varies as the inverse of the radius where the radius is measured from the inner wire where the $B_{1}$ field is perpendicular to a uniformly 
applied $\mathrm{B}_{0}$ field which parallels the axis of the toroid. Using the subject resonator and Rotating Frame Imaging, chemical shifts and distances from the central axis of below $0.1 \mathrm{~mm}$ are simultaneously resolvable. Pressure vessels can be used to place the resonator in a high pressure environment to facilitate measurements under high pressure conditions.

\section{BRIEF DESCRIPTION OF THE DRAWINGS}

The present invention is illustrated in the accompanying drawings where:

Figure 1 is a schematic showing one embodiment of the toroid detector.

Figure 2 illustrates an alternate embodiment of the toroid detector.

Figure 3 show the toroid detector of figure 2 in conjunction with a high pressure reactor body.

\section{DETAILED DESCRIPTION OF THE INVENTION}

Figure 1 depicts the toroid cavity detector 10 . The detector 10 is machined from metal stock to form a toroid cavity resonator. The toroidal 12 portion of the

15 detector 10 is, in the preferred environment, machined from a cylinder of elemental metal or alloy to form a hollow cylinder open at one end and closed at the other. The open end of the toroid 12 is tapped to receive a similarly threaded base 14 . The closed end of the toroid 12 is machined to receive a series of capillary tubes 16 which contain the samples and where the capillary tubes 16 are incrementally spaced on radials originating from the major axis of the toroid 12. A Teflon coated wire 18 is positioned along the major axis of the toroid detector 10 forming a central conductor and is the source for the $B_{1}$ field when the wire 18 is energized to produce 
the $\mathrm{rf}$ field gradient where the field strength is inversely proportional to the radial distance from the central conductor 18 .

Figure 2 represents an alternate embodiment of the toroid detector 20 . In this embodiment 20 , again a cylindrical body 22 is hollowed out where one end of the cylinder 22 closed and the other end is opened. The open end is tapped to receive a similarly threaded base 24 which is attached to the cylindrical body 22 to form a toroid cavity. A Teflon coated wire 26 passes through the base 24 and connects with the closed end of the hollowed out cylinder 22 . The wire 26 is coincidental with the major axis of the cylinder 22 and the long axis of the base 24 slits 27 are cut into the

10 wall and closed end of the cylinder 22 to allow either sample liquids or gasses to flow into the toroid detector 20 . Similarly, slits 28 are cut into the base 24 of the toroid cavity 20 to further permit the flow of gases or liquids through the cavity 20 .

Figure 3 represents a further embodiment of the toroid cavity detector 30 . In a similar manner the cylindrical body 32 of the detector 30 is machined from a 15 cylinder of elemental metal or alloy to form a hollow cylinder closed at one end and open at the opposing end. The open end of the toroid 32 is tapped to receive a similarly threaded base 34 . A Teflon coated wire 36 is positioned along the major axis of the toroid 32 and is threaded at one end to attach it to the closed end of the hollow cylinder. The wire 36 is also oriented along the long axis of the base 34 .

20 When the base 34 is attached to the open end of the hollow cylinder 32 a closed vessel ensues and specimens can be placed in the closed space for testing when the 
central conductor 36 is energized at an $\mathrm{rf}$ frequency to produce a magnetic field $\mathrm{B}_{1}$ whose gradient varies inversely as the radius of the cylinder.

Figure 4 depicts the coupling of a high-pressure Nuclear Magnetic Resonance (NMR) probe and the toroid detector. In this figure, only the embodiment of figure 2 5 is displayed; however, the embodiment of figures 1 or 3 could also be employed in a similar manner with the high-pressure NMR probe. The high-pressure NMR probe is consists of a reactor body 42 in the form of a hollow cylinder with one end opened and the other closed. The closed end of the reactor body 42 is drilled and to receive gas (fluid) connections 44 which are similarly threaded and which serve to link the

10 probe to external sample sources (not shown). The open end of the reactor body 42 is tapped to receive a similarly threaded bottom plug 46 . The bottom plug is also tapped, at one end of the plug 46 , to receive a threaded rf feedthrough 48 and at the other end of the plug to receive the threaded toroid cavity detector 20 base. Both the bottom plug 46 and the rf feedthrough 48 have similarly positioned holes to allow the

15 Teflon coated wire 26 to pass from and external rf source (not shown) to the toroid cavity detector 20 .

The foregoing description of a preferred embodiment of the invention has been presented for purposes of illustration and description. It is not intended to be exhaustive or to limit the invention to the precise form disclosed, and obviously 20 many modifications and variations are possible in light of the above teaching. The embodiments described explain the principles of the invention and practical applications and should enable others skilled in the art to utilize the invention in 
various embodiments and with various modifications as are suited to the particular use contemplated. It is intended that the scope of the invention be defined by the claims appended hereto. 
ABSTRACT

A toroid cavity detection system for determining the spectral properties and distance from a fixed point for a sample using Nuclear Magnetic Resonance. The detection system consists of a toroid with a central conductor oriented along the main axis of the toroidal cylinder and perpendicular to a static uniform magnetic field oriented along the main axis of the toroid. An rf signal is inputted to the central conductor to produce a magnetic field perpendicular to the central axis of the toroid and whose field strength varies as the inverse of the radius of the toroid. The toroid cavity detection system can be used to encapsulate a sample, or the detection system

10 can be perforated to allow a sample to flow into the detection device or to place the samples in specified sample tubes. The central conductor can also be coated to determine the spectral property of the coating and the coating thickness. The sample is then subjected to the respective magnetic fields and the responses measured to determine the desired properties. 


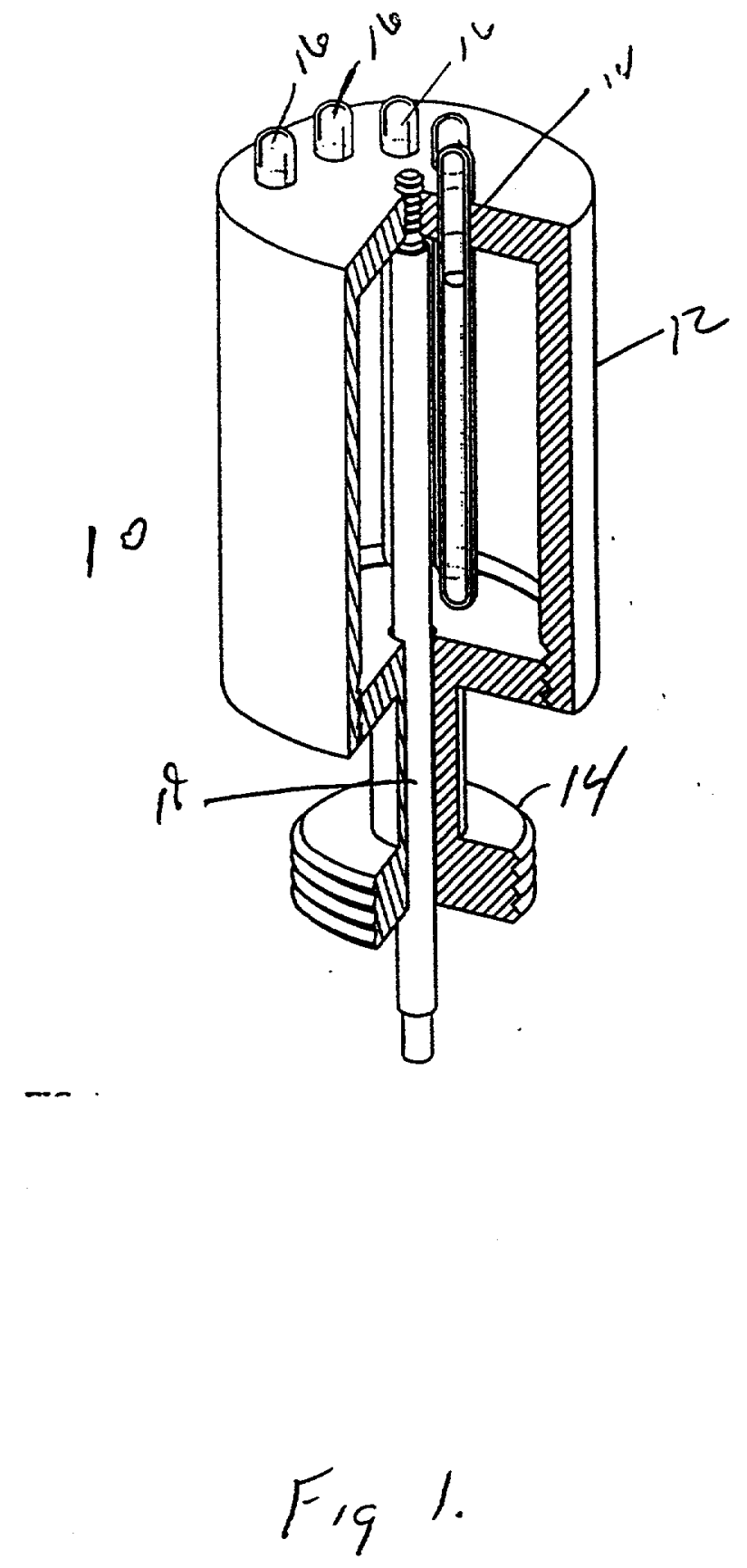




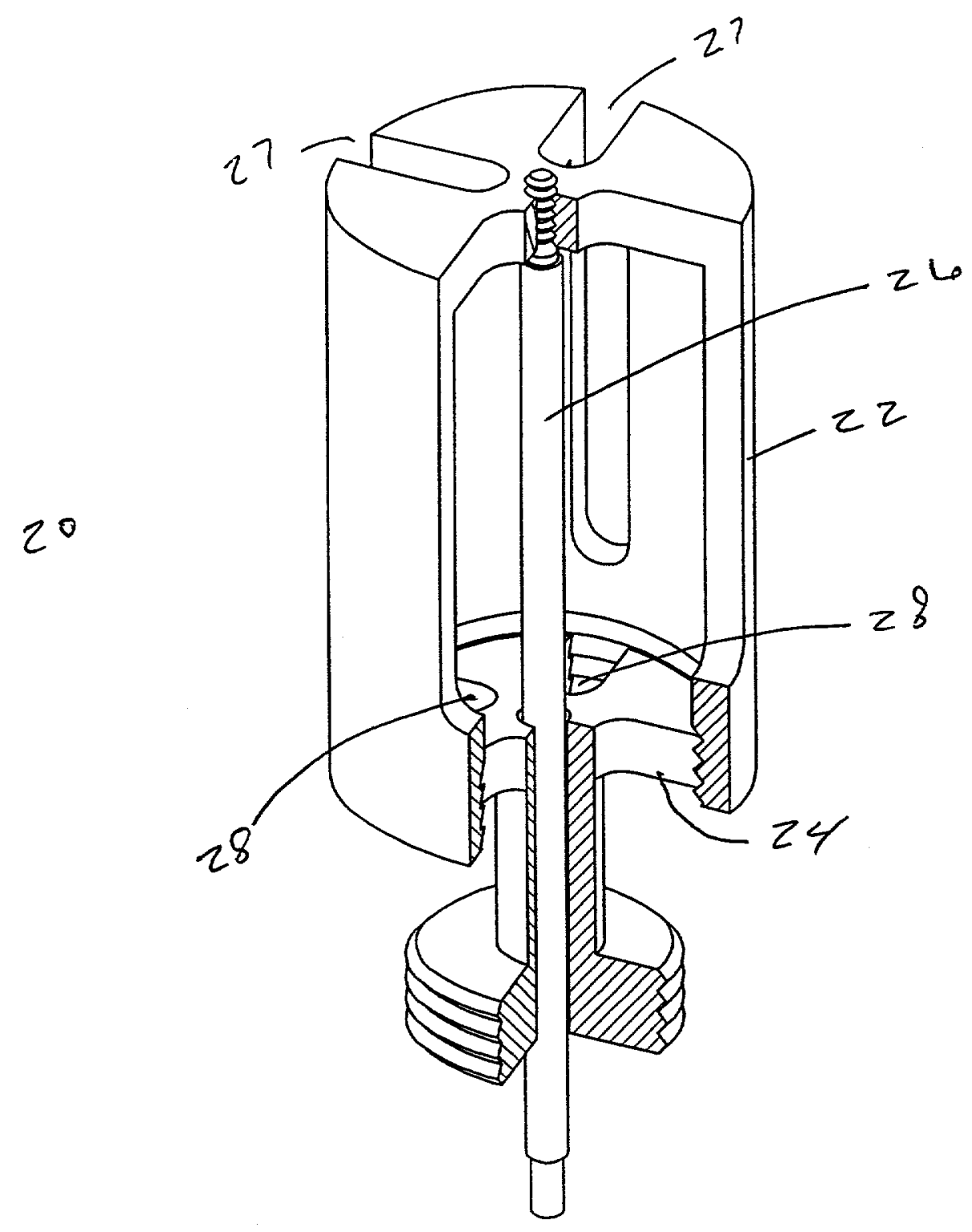

$$
\text { fig } 2
$$




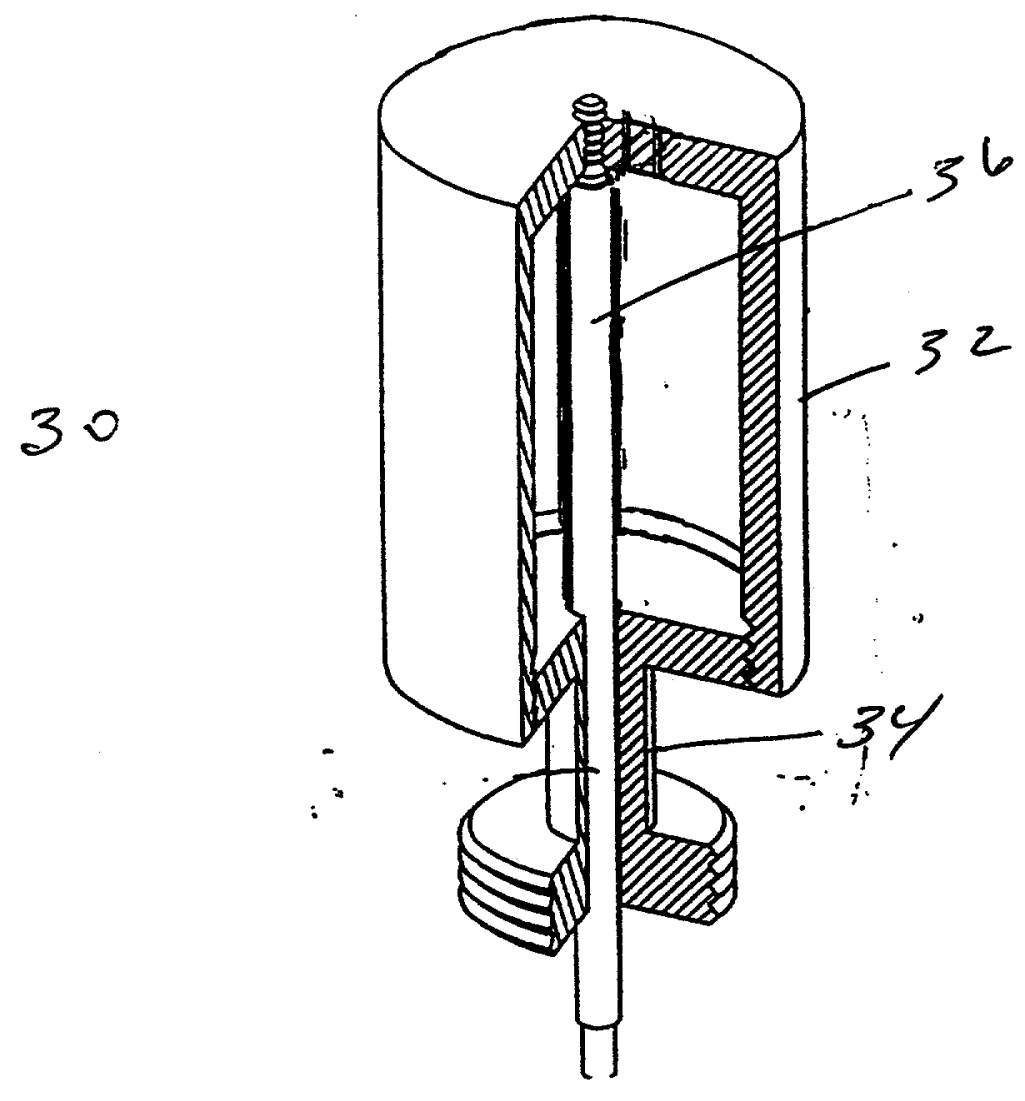

Fig $^{3}$ 


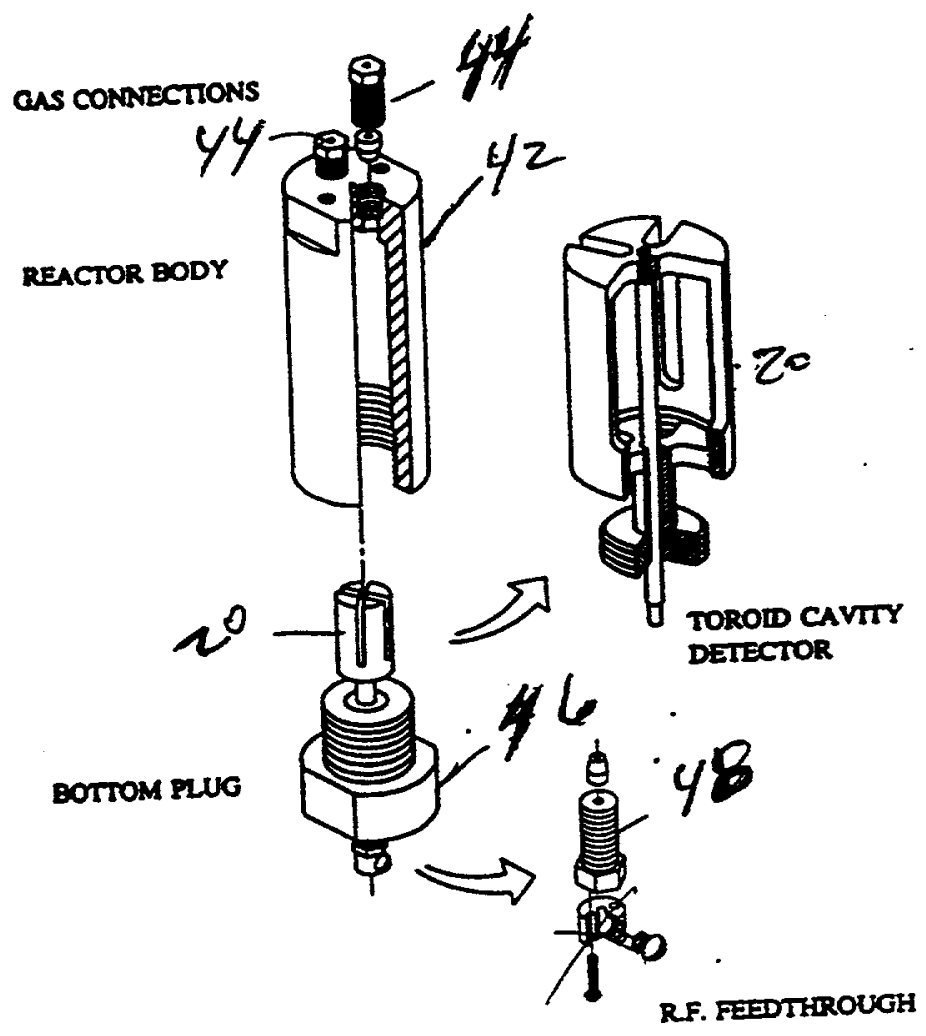

Fig 4. 